\title{
RICKETTSIA FELIS INFECTION IN CAT FLEAS CTENOCEPHALIDES FELIS FELIS
}

\section{Mauricio C. Horta ${ }^{1}$; Fabio B. Scott ${ }^{2}$; Thaís R. Correia ${ }^{2}$; Julio I. Fernandes ${ }^{2}$; Leonardo J. Richtzenhain ${ }^{1}$; Marcelo B. Labruna $^{1^{*}}$}

\begin{abstract}
${ }^{1}$ Departamento de Medicina Veterinária Preventiva e Saúde Animal, Faculdade de Medicina Veterinária, Universidade de São Paulo, São Paulo, SP, Brasil; ${ }^{2}$ Departamento de Parasitologia Animal, Universidade Federal Rural de Rio de Janeiro, Seropédica, RJ, Brasil.
\end{abstract}

Submitted: July 23, 2009; Approved: March 23, 2010

\begin{abstract}
The present study evaluated the rickettsial infection in a laboratory colony of cat fleas, Ctenocephalides felis felis (Bouche) in Brazil. All flea samples (30 eggs, 30 larvae, 30 cocoons, 30 males, and 30 females) tested by polymerase chain reaction (PCR) were shown to contain rickettsial DNA. PCR products, corresponding to the rickettsial $g l t A, h t r A, o m p A$ and $о т p B$ gene partial sequences were sequenced and showed to correspond to Rickettsia felis, indicating that the flea colony was $100 \%$ infected by $R$. felis. The immunofluorescence assay (IFA) showed the presence of $R$. felis-reactive antibodies in blood sera of 7 $(87.5 \%)$ out of 8 cats that were regularly used to feed the flea colony. From 15 humans that used to work with the flea colony in the laboratory, $6(40.0 \%)$ reacted positively to $R$. felis by IFA. Reactive feline and human sera showed low endpoint titers against $R$. felis, varying from 64 to 256 . With the exception of one human serum, all $R$. felis-reactive sera were also reactive to Rickettsia rickettsii and/or Rickettsia parkeri antigens at similar titers to $R$. felis. The single human serum that was reactive solely to $R$. felis had an endpoint titer of 256, indicating that this person was infected by $R$. felis.
\end{abstract}

Key words: Rickettsia felis, flea, Ctenocephalides felis felis, cats, humans

Rickettsia felis, a bacterium formerly belonging to the spotted fever group (SFG) rickettsiae has been recently reclassified into the transitional group (TRG) rickettsiae (9). The organism has been detected infecting fleas (mostly Ctenocephalides spp) in various countries among all continents of the world, except Antarctica (19). R. felis has been reported as the causative agent of flea-borne spotted fever, an emerging human rickettsiosis that has been diagnosed in the United States (27), Mexico (33, 34), Brazil, France (22), Germany (24), Thailand (18), South Korea (7), Tunisia (35), Laos (20),
Spain (5), and Taiwan (29). To date, all cases of human or animal infection by $R$. felis have been confirmed solely by serological and molecular tests; isolation of $R$. felis from humans or any other vertebrate has never been reported.

$R$. felis was first observed within midgut cells of cat fleas, Ctenocephalides felis felis, from a commercial colony maintained by El Labs, Soquel, CA (ELB) in the United Sates (1), which showed $>90 \%$ infection rate, with successful demonstration of transovarial and transstadial transmission as shown by occurrence of infected eggs and unfed larvae (4).

\footnotetext{
*Corresponding Author. Mailing address: FMVZ/USP, Av. Prof. Orlando Marques de Paiva, 87, Cidade Universitária, São Paulo, SP, 05508-270, Brazil. Phone: (11) 3091-1394. Fax: (11) 3091-7028. E-mail: labruna@usp.br
} 
Higgins et al. (10) evaluated the $R$. felis infection in eight laboratory colonies of cat fleas from different areas of USA by molecular analysis, with infection rates varying from 43 to $93 \%$ (mean: $72 \%$ ). Wedincamp \& Foil (30) found $\approx 65 \%$ infection rate in a colony kept at the Lousiania State Agricultural Center St. Gabriel Research Station (LSUSG). In two more recent studies, cats naturally exposed to $R$. felisinfected fleas demonstrated anti-R. felis antibodies $(6,17)$.

The present study aimed to verify Rickettsia felis infection in all developmental stages of the life cycle of $C$. felis felis from a laboratory colony, and to determine the presence of Rickettsia-reactive antibodies in cats and humans in direct contact with the flea colony.

In July 2004, larvae, cocoons, and adults of C. felis felis were obtained from the laboratory flea colony of the Department of Parasitology of the Federal Rural University of Rio de Janeiro, in Seropédica Municipality, Rio de Janeiro, Brazil. In July 2006, flea eggs were also obtained from the same colony. The flea colony has been maintained in the laboratory since 1996. Eight cats were used for feeding adult fleas inside individual cages, in an isolated room. Three times a week, cats were infested with 50 male and 50 female fleas. For colony maintenance, falling eggs from cat fur were collected below the cages, and held inside tubes with artificial diet in an incubator at $27^{\circ} \mathrm{C}$ and $75 \% \mathrm{RH}(8)$.

Samples of 30 eggs, 30 larvae, 30 cocoons, 30 males, and 30 females of $C$. felis felis colony were washed in absolute ethanol and individually submitted to DNA extraction. Each specimen was air dried and subsequently triturated in $50 \mu \mathrm{l}$ of TE buffer [10mM Tris $\mathrm{HCl} ; 1 \mathrm{mM}$ ethylene diamine tetracetic acid (EDTA) $\mathrm{pH}$ 8.0] in sterile micro tubes. The final suspension was boiled at $100^{\circ} \mathrm{C}$ for 20 minutes and held at $20^{\circ} \mathrm{C}$ (11). For every 10 individual flea samples, a blank tube was included in the DNA extraction. Before being triturating for boiling, flea cocoons were individually opened with sterile blades and needles, and only the adult flea present inside the cocoon was used for DNA extraction. This procedure was adopted because in a preliminary assay, whole cocoons from this same flea colony failed to yield PCR products.
Samples were individually tested by polymerase chain reaction (PCR) with a primer pair (CS78 F and CS323 R) targeting a 401-bp fragment of the rickettsial gene gltA (Table 1). Random samples of 2-5 eggs, larvae, cocoons, adult males, and females were submitted to a battery of PCR using all primer pairs described in Table 1, targeting fragments of the rickettsial genes gltA, $h t r A, o m p A$ and $o m p B$, followed by DNA sequencing the PCR products. For each reaction, $5 \mu \mathrm{l}$ of the DNA template from each individual sample (egg, larva, cocoon, male or female) was added to $5 \mu$ of PCR buffer (10X), $8 \mu \mathrm{l}$ of deoxynucleotide triphosphates mixture (1.25mM), $1.5 \mathrm{MgCl}_{2}(50 \mathrm{mM}), 25 \mathrm{pmol}$ of each primer, 0.25 $\mu \mathrm{l}$ of $\mathrm{Taq}$ polymerase $(5,000 \mathrm{U} / \mathrm{ml})$ and bi-distilled water to a final volume of $50 \mu \mathrm{l}$. For each reaction, DNA extracted from Rickettsia parkeri strain At24 was used as positive control (28) and bi-distilled water samples were used as negative controls. PCR thermal conditions were used as previously described (3, 15, 22, 25, 26). PCR products were stained by ethidium bromide and visualized by electrophoresis in $1.5 \%$ agarose gel. For DNA sequencing, PCR products were purified using ExoSap (USB, Cleveland, USA) and sequenced in an automatic sequencer (ABI Prism 3100 Genetic - Applied Biosystems/Perking Elmer, California, USA). All DNA sequences generated in the present study were submitted to BLAST (Basic Local Alignment Search Tool) analysis (2) to determine similarities to other Rickettsia species.

In February 2006, blood serum samples were collected from 8 cats that were used to feed the adult flea colony, and from 15 immune competent humans (researchers, technicians, and students) that had direct contact with these animals and fleas. Sera were tested by Indirect Immunofluorescence Assay (IFA) to detect antibodies reactive to Rickettsia spp. All cats had been continually used for feeding the colony for at least 3 years, while all 15 humans, who had worked with the flea colony for at least 2 years, reported multiple flea bites during this period in the laboratory. IFA was performed in glass slides according to previously described methods $(13,32)$, employing three rickettsial antigens: $R$. felis strain Pedreira (12), Rickettsia rickettsii strain Taiaçú (21), and $R$. parkeri strain At24 (28). 
Human and animal sera were diluted in two-fold increments with PBS starting from a 1:64 dilution. Twenty microliters of diluted sera were added to each well of the antigen slides. The slides were incubated at $37^{\circ} \mathrm{C}$ for 30 minutes in a humid chamber. The slides were rinsed once, and then washed twice for 15 minutes per wash in PBS. The slides were incubated with fluorescein isothiocyanate-labeled goat anti-human IgG or goat anti-cat IgG (Sigma, St Louis, USA) and washed as described earlier. The slides were mounted with buffered glycerin under coverslips, and read using an ultraviolet microscope (Olympus, Tokyo, Japan) at 400x magnification. Serum was considered to contain antibodies against rickettsiae if it displayed a reaction at the 1:64 dilution. Reactive sera were tested in two or three replications before determining the endpoint titer by serial two-fold serum dilution. In each slide, a serum previously shown to be non-reactive (negative control) and a known reactive serum (positive control) were tested.

All the 150 (100\%) flea samples (30 eggs, 30 larvae, 30 cocoons, 30 males, and 30 females) yielded amplicon of the expected length by PCR targeting a 401-bp fragment of the rickettsial gltA gene. PCR product was never visualized in neither blank samples that were submitted to DNA extraction nor PCR negative control samples. Fragments of 1089, 394, 575 and 676-bp of the genes gltA, htrA, ompA and ompB, respectively, were sequenced from each of 2 eggs, 5 larvae, 5 cocoons, 5 adult males, and 5 females. Partial sequences of the gltA, htrA, and ompB genes were $100 \%$ identical to the corresponding sequence of $R$. felis in GenBank (CP000053). Partial sequence of the ompA gene showed a single nucleotide polymorphism (99.8\% of identity) in comparison with $R$. felis in GenBank (CP000053), which resulted in an amino acid change (aspartic acid to tyrosine). Partial ompA sequence of $R$. felis generated in the present study has been submitted to GenBank (accession number FJ425917)

Serum samples from 8 cats and 15 humans were tested against $R$. felis, $R$. rickettsii and $R$. parkeri antigens by IFA. Six humans $(40.0 \%)$ and 7 cats $(87.5 \%)$ showed titers $\geq 64$ to at least one Rickettsia species (Table 2). All samples reactive to $R$. rickettsii or $R$. parkeri were also reactive to $R$. felis. One human serum was reactive solely to $R$. felis.

Table 1. Primers used for amplification of rickettsial genes in both PCR and DNA sequencing.

\begin{tabular}{|c|c|c|c|c|}
\hline $\begin{array}{c}\text { Primer } \\
\text { Pairs }\end{array}$ & Gene & Nucleotide sequence (5'-3') & $\begin{array}{c}\text { Expected } \\
\text { amplicon } \\
\text { length* }\end{array}$ & Reference \\
\hline CS78 F & \multirow{2}{*}{ gltA } & GCAAGTATCGGTGAGGATGTAAT & \multirow{2}{*}{$401 \mathrm{bp}$} & 15 \\
\hline CS323 R & & GCTTCCTTAAAATTCAATAAATCAGGAT & & 15 \\
\hline $\mathrm{CS} 239 \mathrm{~F}$ & \multirow{2}{*}{ gltA } & GCTCTTCTCATCCTATGGCTATTAT & \multirow{2}{*}{834 bp } & 15 \\
\hline CS1069 R & & CAGGGTCTTCGTGCATTTCTT & & 15 \\
\hline $17 \mathrm{~K} 1 \mathrm{~F}$ & \multirow{2}{*}{$h t r A$} & GCTCTTGCAACTTCTATGTT & \multirow{2}{*}{$434 \mathrm{bp}$} & 3 \\
\hline $17 \mathrm{~K} 2 \mathrm{R}$ & & CATTGTTCGTCAGGTTGGCG & & 3 \\
\hline $190.70 \mathrm{~F}$ & \multirow{2}{*}{ ompA } & ATGGCGAATATTTCTCCAAAA & \multirow{2}{*}{$617 \mathrm{bp}$} & 26 \\
\hline $190.701 \mathrm{R}$ & & GTTCCGTTAATGGCAGCATCT & & 25 \\
\hline $190.59 \mathrm{~F}$ & \multirow{2}{*}{ oтp B } & CCGCAGGGTTGGTAACTGC & \multirow{2}{*}{862 bp } & 22 \\
\hline $190.807 \mathrm{R}$ & & CCTTTTAGATTACCGCCTAA & & 22 \\
\hline
\end{tabular}

* according $R$. felis (strain URRWXCal2) sequence in GenBank (CP000053) 
Table 2. Endpoint titers of indirect immunofluorescence assay (IFA) for Rickettsia felis, Rickettsia rickettsii and Rickettsia parkeri in cats and humans with direct contact with a Rickettsia felis-infected laboratory flea colony.

\begin{tabular}{|c|c|c|c|}
\hline \multirow{2}{*}{ Sample } & \multicolumn{3}{|c|}{ Rickettsia antigens used for IFA } \\
\hline & R. felis & R. rickettsii & R. parkeri \\
\hline Cat 1 & 64 & 64 & 64 \\
\hline Cat 2 & 64 & - & 64 \\
\hline Cat 3 & 128 & 128 & 128 \\
\hline Cat 4 & 64 & - & 64 \\
\hline Cat 5 & 64 & 64 & 64 \\
\hline Cat 6 & 64 & 64 & 128 \\
\hline Cat 7 & 128 & 64 & 64 \\
\hline Cat 8 & - & - & - \\
\hline Human 1 & - & - & - \\
\hline Human 2 & - & - & - \\
\hline Human 3 & - & - & - \\
\hline Human 4 & 64 & 128 & 64 \\
\hline Human 5 & - & - & - \\
\hline Human 6 & - & - & - \\
\hline Human 7 & - & - & - \\
\hline Human 8 & 128 & 256 & - \\
\hline Human 9 & - & - & - \\
\hline Human 10 & 64 & 128 & 128 \\
\hline Human 11 & 256 & - & - \\
\hline Human 12 & - & - & - \\
\hline Human 13 & 128 & - & 128 \\
\hline Human 14 & - & - & - \\
\hline Human 15 & 128 & 256 & 256 \\
\hline
\end{tabular}

- : non reactive at titer $\geq 64$

In the present study, DNA sequencing of PCR products from random samples indicate that all individuals of the flea colony were infected by $R$. felis. High infection rates, varying from 43 to $100 \%$, were also reported for laboratory cat flea colonies in the United States (23). Previous studies with wild cat flea populations in South America have reported R. felisinfection rates varying from 13.5 to $90 \%$ (17). Since we found $R$. felis DNA in eggs, and all post-embryonic developmental stages of cat fleas, our results corroborate previous studies showing that $R$. felis is transmitted transovarially and transstadially in cat fleas $(4,31)$.

In a recent study (17) with cats naturally infested by $R$. felis-infected cat fleas (70\% infection rate) in Chile, 16 $(72.7 \%)$ out of 22 cats had R. felis-reactive antibodies (titer $\geq 64)$. Among those, 3 (13.6\%) also reacted with $R$. parkeri and $2(9.1 \%)$ with $R$. rickettsii. Like in the present study, no Chilean cat serum reacted with $R$. rickettsii or $R$. parkeri without reacting with $R$. felis. $R$. felis-endpoint titers (128 to 512) in four of these cats were at least 4-fold higher than that to any of the other Rickettsia species, suggesting homologous reaction to $R$. felis infection (17). In the present study, 7 out of 8 cats used to feed a R. felis-infected cat flea colony $(100 \%$ infection rate) showed antibodies reactive to both $R$. felis and $R$. parkeri antigens, and 5 cats were also seroreactive to $R$. rickettsii antigens. Endpoint titers to the three rickettsial antigens were always low, varying from 64 to 128 (Table 2). In this case, no cat showed endpoint titer 4-fold higher for a Rickettsia species than for the other species. However, since these cats have been continually infested by $R$. felis-infected cat fleas in the laboratory, under no contact with ticks or lice (potential vectors of other Rickettsia species) and fed exclusively with commercial pellets and water (cats were not allowed to go out hunting), it is likely that their serological reactiveness to SFG Rickettsia was elicited by $R$. felis during 
previous contacts with infected cat fleas from the colony.

Since all 15 humans sampled in the present study had recent history of multiple flea bites while handling the flea colony in the laboratory, we expected most, or even all, of them to show anti- $R$. felis antibodies, since it has been shown that $R$. felis is a human pathogen (19). However, only $6(40 \%)$ human sera were reactive to $R$. felis antigens. Five of these sera also showed similar antibody titers to $R$. rickettsii and/or $R$. parkeri, precluding any inference of the rickettsial agent responsible for eliciting serological response, because they all lived outside the laboratory and had different histories of exposure to additional rickettsial vectors, especially ticks (data not shown). On the other hand, one human serum (human 11, Table 2) was reactive to $R$. felis (titer 256) without reacting with the remaining rickettsial antigens. According to serological interpretations used in previous studies $(14,16,18$, $29)$, this human 11 was infected by $R$. felis or a very closely related genotype because its endpoint titer to $R$. felis was at least 4-fold higher than that to any of the other Rickettsia species. Although recent febrile illness compatible with classic spotted fever (fever, headache, and rash) was not recalled by any of the 15 humans (data not shown), rickettsiosis can manifest with a multiplicity of general symptoms (19), making it difficult to recall previous clinical rickettsiosis with certainty.

$R$. felis is currently recognized as an emerging human pathogen of worldwide distribution However, the global scarcity of $R$. felis clinical reports deserves further attention. As the disease is considered to be rare, its confirmatory diagnostic testing is difficult because very few laboratories are equipped with specific diagnostic assays. These facts should be related to the low number of flea-borne rickettsioses cases that have been confirmed in the world.

\section{ACKNOWLEDGMENTS}

This work was supported by Fundação de Amparo à Pesquisa do Estado de São Paulo - FAPESP (grants to M.C.H., and M.B.L.) and Conselho Nacional de Desenvolvimento Científico e tecnológico - CNPq (scientific career scholarship to M.B.L.).

\section{REFERENCES}

1. Adams, J.R.; Schmidtmann, E.T.; Azad, A.F. (1990). Infection of colonized cat fleas, Ctenocephalides felis (Bouché), with a rickettsia-like microorganism. Am. J. Trop. Med. Hyg. 43, 400-409.

2. Altschul, S.F.; Gish, W.; Miller, W.; Myers, E.W.; Lipman, D.J. (1990). Basic local alignment search tool. J. Mol. Biol. 215, 403-410.

3. Azad, A.F.; Webb, L.; Carl, M.; Dasch, G.A. (1990). Detection of Rickettsiae in arthropod vectors by DNA amplification using the polymerase chain reaction. Ann. N. Y. Acad. Sci. 590, 557-563.

4. Azad, A.F.; Sacci Jr, J.B.; Nelson, W.M.; Dasch, G.A.; Schmidtmann, E.T.; Carl, M. (1992). Genetic characterization and transovarial transmission of a typhus-like rickettsia found in cat fleas. Proc. Natl. Acad. Sci. USA 89, 43-46.

5. Bernabeu-Wittel, M.; Del Toro, M.D.; Nogueras, M.M.; Muniain, M.A.; Cardenosa, N.; Márquez, F.J.; Segura, F.; Pachón, J. (2006). Seroepidemiological study of Rickettsia felis, Rickettsia typhi, and Rickettsia conorii infection among the population of southern Spain. Eur. J. Clin. Microbiol. Infect. Dis. 25, 375-381.

6. Case, J.B.; Chomel, B.; Nicholson, W.; Foley, J.E. (2006). Serological survey of vector-borne zoonotic pathogens in pet cats and cats from animal shelters and feral colonies. J. Feline Med. Surg. 8, 111-117.

7. Choi, Y.J.; Jang, W.J.; Ryu, J.S.; Lee, S.H.; Park, K.H.; Paik, H.S.; Koh, Y.S.; Choi, M.S.; Kim, I.S. (2005). Spotted fever group and typhus group rickettsioses in humans, South Korea. Emerg. Infect. Dis. 11, 237-244.

8. Correia, T.R.; Souza, C.P.; Fernandes, J.I.; Martins, I.V.F.; Santos, H.D.; Scott, F.B. (2003). Ciclo biológico de Ctenocephalides felis felis (Bouché, 1835) (Shiphonaptera:Pulididae) a partir de diferentes dietas artificiais. Rev. Bras. Zoocien., Juiz de Fora 5, 153-160.

9. Gillespie, J.J.; Beier; M.S.; Rahman, M.S.; Ammerman, N.C.; Shallom, J.M.; Purkayastha, A.; Sobral, B.S.; Azad, A.F. (2007). Plasmids and rickettsial evolution: insight from Rickettsia felis. PLOS ONE 2, e266.

10. Higgins, J.A.; Sacci Jr, J.B.; Schriefer, M.E.; Endris, R.G.; Azad, A.F. (1994). Molecular identification of rickettsia-like microorganisms associated with colonized cat fleas (Ctenocephalides felis). Insect Molecular Biol. 3, 27-33.

11. Horta, M.C.; Pinter, A.; Cortez, A.; Soares, R.M.; Gennari, S.M.; Schumaker, T.T.S.; Labruna, M.B. (2005). Rickettsia felis (Rickettsiales:Rickettsiaceae) in Ctenocephalides felis felis (Siphonaptera: Pulicidae) in the State of São Paulo, Brazil. Arq. Bras. Méd. Vet. Zootec. 57, 321-325.

12. Horta, M.C.; Labruna, M.B.; Durigon, E.L.; Schumaker, T.T.S. (2006). Isolation and Cultivation of Rickettsia felis in the mosquito cell line C6/36. Appl. Environ. Microbiol. 72, 1705-1707.

13. Horta, M.C.; Labruna, M.B.; Pinter, A.; Linardi, P.M.; Schumaker, T.T.S. (2007). Rickettsia infection in five areas of the State of São Paulo, Brazil. 
Mem. Inst. Oswaldo Cruz 102, 793-801.

14. Jansen, A.; La Scola, B.; Raoult, D.; Lierz, M.; Wichmann, O.; Stark, K.; Schneider, T. (2008). Antibodies against Rickettsia spp. in hunters, Germany. Emerg. Infect. Dis. 14, 1961-1963.

15. Labruna, M.B.; Whitworth, T.; Horta, M.C.; Bouyer, D.H.; McBride, J.W.; Pinter, A.; Popov, V.; Gennari, S.M.; Walker, D.H. (2004). Rickettsia species infecting Amblyomma cooperi ticks from an area in the State of São Paulo, Brazil, where Brazilian spotted fever is endemic. $J$. Clin. Microbiol. 42, 90-98.

16. Labruna, M.B.; Horta, M.C.; Aguiar, D.M.; Cavalcante, G.T.; Pinter, A.; Gennari, S.M.; Aranha, L.M.A. (2007). Prevalence of Rickettsia infection in dogs from the urban and rural areas of Mone Negro Municipality, Western Amazon, Brazil. Vector-Borne Zoonotic Dis. 7, 249-255.

17. Labruna, M.B.; Ogrzewalska, M.; Moraes-Filho, J.; Lepe, P.; Gallegos, J.L.; López, J. (2007). Rickettsia felis in Chile. Emerg Infect Dis. 13, 1794-1795.

18. Parola, P.; Miller, R.S.; McDaniel, P.; Telforf III, S.R.; Rolain, J.M.; Wongsrichanalai, C.; Raoult, D. (2003). Emerging rickettsioses of the Thai-Myanmar Border. Emerg. Infect. Dis. 9, 592-595.

19. Pérez-Osorio, C.E.; Zavala-Velazquez, J.E.; León, J.J.A.; Zavala-Castro, J.E. (2008). Rickettsia felis as emergent global threat for humans. Emerg. Infect. Dis. 14, 1019-1023.

20. Phongmany, S.; Rolain, J.M.; Phetsouvanh, R.; Blacksell, S.D.; Soukkhaseum, V.; Rasachack, B.; Phiasakha, K.; Soukkhaseum, S.; Frichithavong, K.; Chu, V.; Keolouangkhot, V.; Martinez-Aussel, B.; Chang, K.; Darasavath, C.; Rattanavong, O.; Sisouphone, S.; Mayxay, M.; Vidamaly, S.; Parola, P.; Thammavong, C.; Heuangvongsy, M.; Syhavong, B.; Raoult, D.; White, N.J.; Newton, P.N. (2006). Rickettsial infections and fever, Vientiane, Laos. Emerg. Infect. Dis. 12, 256-262.

21. Pinter, A.; Labruna, M.B. (2006). Isolation of Rickettsia rickettsii and Rickettsia bellii in cell culture from the tick Amblyomma aureolatum in Brazil. Ann. N. Y. Acad. Sci. 1078, 523-529.

22. Raoult, D.; La Scola, B.; Enea, M.; Fournier, P.E.; Roux, V.; Fenollar, F.; Galvão, M.A.M.; Lamballerie, X. (2001). A flea-associated Rickettsia pathogenic for humans. Emerg. Infec. Dis. 7, 73-81

23. Reif, K.E.; Stout, R.W.; Henry, G.C.; Foil, L.D.; Macaluso, K.R. (2008). Prevalence and infection load dynamics of Rickettsia felis in actively feeding cat fleas. PLoS ONE 3, e2805.

24. Richter, J.; Fournier, P.E.; Petridou, J.; Hãussinger, D.; Raoult, D. (2002).
Rickettsia felis infection acquired in Europe and documented by by polymerase chain reaction. Emerg. Infect. Dis. 8, 207-208.

25. Roux, V.; Fournier, P.E.; Raoult, D. (1996). Differentiation of spotted fever group rickettsiae by sequencing and analysis of restriction fragment length polymorphism of PCR amplified DNA of the gene encoding the protein rOmpA. J. Clin. Microbiol. 34, 2058-2065.

26. Roux, V. (1999). Plylogenetic analysis and taxonomic relationship among the genus Rickettsia. In: Raoult, D.; Brouqui, P. (eds). Rickettsiae and rickettsial diseases at the turn of the third millennium. Elsevier, Marseille, France, p. 52-66.

27. Schriefer, M.E.; Sacci, J.B.; Dumler, J.S.; Bullen, M.G.; Azad, A.F. (1994). Identification of a novel rickettsial infection in a patient diagnosed with murine typhus. J. Clin. Microbiol. 32, 949-954.

28. Silveira, I.; Pacheco, R.C.; Szabó, M.P.J.; Ramos, H.G.C.; Labruna, M.B. (2007). First report of Rickettsia parkeri in Brazil. Emerg. Infect. Dis. 13, 1111-1113.

29. Tsai, K.H.; Lu, H.Y.; Tsai, J.J.; Yu, S.K.; Huang, J.H.; Shu, P.Y. (2008). Human case of Rickettsia felis infection, Taiwan. Emerg. Infect. Dis. 14, 1970-1972.

30. Wedincamp, J.; Foil, L.D. (2000). Infection and seroconversion of cats exposed to cat fleas (Ctenocephalides felis Bouché) infected with Rickettsia felis. J. Vector Ecology 25, 123-126.

31. Wedincamp, J.; Foil, L.D. (2002). Vertical transmission of Rickettsia felis in the cat flea (Ctenocephalides felis Bouché). J. Vector Ecology 27, 96101.

32. Zavala-Velazquez, J.E.; Yu, X.J.; Walter, D.H. (1996). Unrecognized spotted fever group rickettsiosis masquerading as dengue fever in Mexico. Am. J. Trop. Med. Hyg. 55, 157-159.

33. Zavala-Velazquez, J.E.; Ruiz-Sosa, J.A.; Sánchez-Elias, R.A.; BecerraCarmona, G.; Walker, D.H. (2000). Ricketsia felis rickettsiosis in Yucatán. Lancet 356, 1079-1080.

34. Zavala-Velazquez, J.E.; Laviada-Molina, H.; Zavala-Castro, J.; PerezOsorio, C.; Becerra-Carmona, G.; Ruiz-Sosa, J.A.; Bouyer, D.H.; Walker, D.H. (2006). Rickettsia felis, the agent of an emerging infectious disease: report of a new case in Mexico. Arch. Med. Research 37, 419-422.

35. Znazen, A.; Rolain, J.M.; Hammami, N.; Hammami, A.; Jemaa, M.B.; Raoult, D. (2006). Rickettsia felis infection, Tunisia. Emerg. Infect. Dis. $12,138-140$. 